\title{
Indium oxide thin film based ammonia gas and ethanol vapour sensor
}

\author{
K K MAKHIJA*, ARABINDA RAY ${ }^{\dagger}$, R M PATEL ${ }^{\dagger}$, U B TRIVEDI \\ and $\mathrm{H}$ N KAPSE \\ Department of Electronics, ${ }^{\dagger}$ Department of Chemistry, Sardar Patel University, Vallabh Vidyanagar 388 120, India \\ ${ }^{\ddagger}$ Pramukhswami Computer Institute, Vallabh Vidyanagar 388 120, India
}

MS received 29 September 2003; revised 19 February 2004

\begin{abstract}
A sensor for ammonia gas and ethanol vapour has been fabricated using indium oxide thin film as sensing layer and indium tin oxide thin film encapsulated in poly(methyl methacrylate) (PMMA) as a miniature heater. For the fabrication of miniature heater indium tin oxide thin film was grown on special high temperature corning glass substrate by flash evaporation method. Gold was deposited on the film using thermal evaporation technique under high vacuum. The film was then annealed at $700 \mathrm{~K}$ for an hour. The thermocouple attached on sensing surface measures the appropriate operating temperature.

The thin film gas sensor for ammonia was operated at different concentrations in the temperature range 323-493 K. At $473 \mathrm{~K}$ the sensitivity of the sensor was found to be saturate. The detrimental effect of humidity on ammonia sensing is removed by intermittent periodic heating of the sensor at the two temperatures 323K and $448 \mathrm{~K}$, respectively. The indium oxide ethanol vapour sensor operated at fixed concentration of $400 \mathrm{ppm}$ in the temperature range 293-393 K. Above $373 \mathrm{~K}$, the sensor conductance was found to be saturate. With various thicknesses from $150-300 \mathrm{~nm}$ of indium oxide sensor there was no variation in the sensitivity measurements of ethanol vapour. The block diagram of circuits for detecting the ammonia gas and ethanol vapour has been included in this paper.
\end{abstract}

Keywords. Indium oxide; ammonia sensor; ethanol vapour sensor; PMMA and Au.

\section{Introduction}

Gas sensors play vital role in detecting, monitoring and controlling the presence of hazardous and poisonous gases in the atmosphere at very low concentrations. Semiconductor gas sensors in the form of thin films are highly sensitive and reliable, having a performance/price ratio comparable to that of microelectronic components. Polycrystalline tin oxide based gas sensors are commercially important for detecting reducible gases (Gopel 1985) such as hydrogen, methane, butane and carbon monoxide with high sensitivities. It is well known that the gas adsorption onto the surface of a semiconductor can influence its electrical conductivity. In fact, conductivity of semiconductor gas sensor changes by few orders of magnitude with respect to initial value in the presence of gas concentrations up to few ppm in air at ambient pressure.

The electrolytic techniques using diaphragm electrodes are generally used for the detection of ammonia. However, this method is expensive and does not have sufficient sensitivity or selectivity for ammonia (Nanto et al 1986; Moseley and Williams 1990). Palladium gate metal oxide semiconductor (MOS) device is sensitive to

\footnotetext{
*Author for correspondence (kkmakhija2000@indiatimes.com)
}

ammonia gas, but the device does not have sufficient selectivity. Semiconducting oxide sensors such as $\mathrm{ZnO}$ (Nanto et al 1986), $\mathrm{SnO}_{2}$ (Kanefusa et al 1983), $\mathrm{TiO}_{2}$ (Moseley and Williams 1990) with additives (e.g. Al, $\mathrm{Cr}_{2} \mathrm{O}_{3}$ ) are also used as ammonia sensors. $\mathrm{MoO}_{3} / \mathrm{TiO}_{2}$ and $\mathrm{Bi}_{2} \mathrm{MoO}_{6}$ have been employed as ammonia sensors (Raju and Rao 1991). Commercially available FIGARO gas sensor No. TGS 824 can detect ammonia gas in the range of $30-300 \mathrm{ppm}$.

The most commonly used efficient ethanol sensors are based on $\mathrm{LaNiO}_{3}$ (Obayashi et al 1976), ZnO (Raju and Rao 1991), $\mathrm{SnO}_{2}$ (Ogawa et al 1981; Sears et al 1989; Oomman et al 1998; Tadashi 1998). It is also well known that electrical properties of semiconducting oxides like $\mathrm{InO}_{x}$ depend strongly on defect density created by either external doping or distributed stoichiometry as well as their growth conditions. Recently a sufficient amount of work has been reported on materials like $\mathrm{In}_{2} \mathrm{O}_{3}$ ( $\mathrm{Rao}$ et al 1992; Fritzche et al 1994; Girtan and Rusu 1999-2000; Gagaoudakis et al 2001) and indium tin oxide (Manifacier et al 1979; Hamberg et al 1984; Kim et al 1990; Echigoya et al 1992) to detect hydrocarbon gases, ozone, nitric oxide gases etc. But no work has been reported on the indium oxide to detect ammonia gas and ethanol vapours at low temperature. We report here the use of indium oxide thin film gas sensors to sense ammonia gas and ethanol vapours at relatively low temperature. 
We have prepared indium oxide $\left(\mathrm{In}_{2} \mathrm{O}_{3}\right)$ films with different crystallites by changing substrate temperature and deposition rates in the thermal evaporation technique under high vacuum. Changes in the stoichiometry of film provided variety of defect densities. When semiconductor materials are used as gas sensors, either deposited as thin films or thick films or sintered powder, they are found to detect a wide variety of gases. There are two stages of gas sensing effect:

- Atmospheric oxygen gets adsorbed on the surface and removing a carrier from the conduction band of $n$-type semiconductor gas sensors, becomes, $\mathrm{O}^{-2}$ or $\mathrm{O}^{-}$species. This phenomenon reduces the overall conductance.

- The reducing agents, such as hydrogen, carbon monoxide, ammonia and hydrocarbon gases will react with chemi-adsorbed oxygen. This process will reinject the carrier and increase the sensor conductance.

It is well known that the electrical conductivity in indium oxide is due to non-stoichiometric composition as a result of oxygen deficiency. The conductivity is of $n$-type. When the indium oxide sensor surface is placed in an air ambient, the oxygen molecules are adsorbed at the surface resulting in the formation of $\mathrm{O}_{2}^{-}, \mathrm{O}^{-}, \mathrm{O}^{2-}$ ions, thus decreasing the concentration of the number of charge carriers near the surface giving rise to a depletion region. When exposed to reducing gases like ammonia and ethanol vapour, mutual interaction between the reactant i.e. reducing gas and oxygen species, results in oxidation of reducing gas at the surface (Ertl 1982). This oxidation phenomenon helps in the removal of oxygen ion from indium oxide surface resulting in decrease in the barrier height, thus increasing the conductance. During the chemisorption at higher temperature $400 \mathrm{~K}$, oxygen is adsorbed in ionic form as shown in the following reactions

$$
\begin{aligned}
& \mathrm{O}_{2}+e^{-}=\mathrm{O}_{2}^{-}, \\
& \mathrm{O}_{2}^{-}+e^{-}=2 \mathrm{O}^{-} .
\end{aligned}
$$

Above $450 \mathrm{~K}$, the reactivity of $\mathrm{O}^{-2}$ species is high (Nelli and Sbervegliere 1994). The formation of $\mathrm{O}^{-2}$ species is also possible as follows

$$
\mathrm{O}_{\mathrm{ads}}^{-}+e^{-}=\mathrm{O}^{-2} \text {. }
$$

$\mathrm{O}^{-2}$ ions are not adsorbed because these species are not stable and are usually trapped by oxygen vacancies.

The present investigation aims to fabricate chemical gas sensing device with good sensitivity that can operate at low temperature. The gas sensor fabricated for this study uses indium oxide $\left(\mathrm{In}_{2} \mathrm{O}_{3}\right.$ phase $)$ as sensing material and indium tin oxide film encapsulated in poly (methyl methacrylate) (PMMA) as miniature heater.

\section{Experimental}

\subsection{Fabrication of thin film gas sensors}

Indium oxide thin films were grown on alumina substrates using thermal evaporation, followed by annealing in humid environment. The optimized growth parameters reported by Patel et al $(1994,1995)$ and Patel and Lashkari (1992) to achieve the best polycrystalline indium tin oxide thin films were utilized for the growth of indium oxide thin films. The optimized growth parameters and their results are illustrated in table 1 . In order to achieve desirable film properties, cleaning of the substrate surface prior to film deposition is essential. For this the glass substrates were cleaned by successive immersion in chromic acid and doubly distilled water, and were then ultrasonically cleaned in a detergent solution. After washing with double distilled water, they were rinsed with acetone and dried under an electric lamp (100 W). Thin films of indium oxide were grown using direct thermal evaporation technique. The source material was a fine powder of indium oxide (Aldrich). Vacuum of the order of $10^{-4} \mathrm{~Pa}$ was maintained during deposition of films. The films were grown at different substrate temperatures ranging from $300-573 \mathrm{~K}$. A flat electric heater was used for substrate heating. The substrate temperature was monitored using chromel-alumel thermocouple mounted on the surface of the substrates. The distance of about $20 \mathrm{~cm}$ was kept constant between tantalum boat and substrate. After maintaining a steady substrate temperature, the electrical supply to the boat was switched on and was gradually increased so that temperature rose to about $1100 \mathrm{~K}$. The evaporated material then got deposited on the substrate. The thickness of the films was measured by the quartz crystal monitor, which was mounted near to the substrate holder. The rate of deposition of the film was kept constant at about $3-5 \mathrm{~nm} / \mathrm{s}$. The indium oxide thin films of various thicknesses of about $100-300 \mathrm{~nm}$ were grown on alumina substrates (total surface area, $5 \times 5 \mathrm{~mm}$, $250 \mu$ thickness). Gold particles were distributed finely on the top of indium oxide film by thermal evaporation under high vacuum to form a film of thickness 20-30 A. This Au layer acts as a stimulating layer. The films so obtained were annealed at $700 \mathrm{~K}$ for $1 \mathrm{~h}$ in $80 \%$ relative humidity. Two thin film gold electrodes were also depo-

Table 1. Parameters for fabrication of indium oxide thin film and its characteristic properties.

\begin{tabular}{lc}
\hline A. Fabrication parameters & \\
High vacuum & $10^{-4}$ pascal \\
Thickness of films & $100-300 \mathrm{~nm}$ \\
Substrate temperature & $573 \mathrm{~K}$ \\
Post deposition annealing at & $700 \mathrm{~K}$ for an hour \\
Relative humidity & $85 \%$ \\
B. Characteristic properties & \\
Electrical resistivity & $1.12 \times 10^{-3} \Omega / \mathrm{cm}$ \\
Carrier concentration, $\eta$ & $4.2 \times 10^{20} \mathrm{~cm}^{-3}$ \\
Carrier mobility, $\mu$ & $55 \mathrm{~cm}^{2} / \mathrm{V}-\mathrm{s}$ \\
Type of conductivity & $\mathrm{N}$ \\
Optical transparency & $65 \%$ \\
Optical band gap (direct) & $3.62 \mathrm{eV}$ \\
\hline
\end{tabular}


sited at each end of indium oxide thin films for ohmic contacts to permit electrical measurements.

Post deposition annealing of indium oxide in the furnace at different values of relative humidity was carried out to improve the electrical conductivity and transparency. The detailed procedure for deposition of indium tin oxide films and the effect of annealing in humid air on electrical properties are reported elsewhere (Patel and Lashkari 1992). The annealing temperature was kept constant at about $700 \mathrm{~K}$.

\subsection{Miniature heater}

Indium tin oxide was used for the fabrication of miniature heater and the following processes were carried out: Indium tin oxide thin films of 300-400 nm were grown on special high temperature borosilicate corning glasses by flash evaporation in vacuum of $10^{-4} \mathrm{~Pa}$. The optimized growth parameters and their results are shown in table 2 . The source material was a fine thin powder of indium tin oxide $\left(\mathrm{In}_{2} \mathrm{O}_{3}+17 \% \mathrm{SnO}_{2}\right.$, Alfa Johnson Matthey) kept in feeder which was vibrated by electromagnet, to let powder drop slowly on tantalum boat. After maintaining substrate temperature of $673 \mathrm{~K}$ in vacuum of $10^{-4} \mathrm{~Pa}$, the electrical supply to the electromagnetic vibrator was switched on. Gradually the fine grains of indium tin oxide powder could fall on the heated tantalum boat and evaporate instantaneously. The film thickness was maintained at around $300-400 \mathrm{~nm}$ for all sets of miniature heater fabrication. The size of indium oxide miniature heater is $2 \times 1 \mathrm{~cm}$ of total surface area.

Row type parallel configuration of indium tin oxide thin films obtained by flash evaporation technique was used as miniature heaters. Due to their reduced sensitivity to Na-ion migration and lesser likelihood of failure under humidity and load conditions, simple straight-line shape was preferred to meandering patterns of indium tin oxide heater.

Table 2. Parameters for fabrication of indium tin oxide miniature heater and its characteristics properties.

\begin{tabular}{lc}
\hline A. Fabrication parameters & \\
High vacuum & $10^{-4}$ pascal \\
Thickness of films & $300-400 \mathrm{~nm}$ \\
Substrate temperature & $673 \mathrm{~K}$ \\
Post deposition annealing at & $700 \mathrm{~K}$ for an hour \\
Relative humidity & $85 \%$ \\
& \\
B. Characteristic parameters & \\
Electrical resistivity & $5 \cdot 6 \times 10^{-4} \Omega / \mathrm{cm}$ \\
Carrier concentration, $\eta$ & $4 \times 10^{20} \mathrm{~cm}^{-3}$ \\
Carrier mobility, $\mu$ & $35 \mathrm{~cm}^{2} / \mathrm{V}-\mathrm{s}$ \\
Type of conductivity & $\mathrm{N}$ \\
Optical transparency & $75 \%$ \\
Optical band gap (direct) & $3.68 \mathrm{eV}$ \\
\hline
\end{tabular}

PMMA powder (molecular weight, $15000 \mathrm{amu}$ ) with glass transition temperature of $378 \mathrm{~K}$ and melting temperature of $498 \mathrm{~K}$ was dissolved in dimethyl formide (DMF) at concentrations of $20 \%$ and $25 \%$ (wt/vol.). PMMA layers of various thicknesses were applied on device by spin coating the solution at speeds of 1000-2000 rpm for $1 \mathrm{~h}$. Multiple spin coating was used to obtain film thickness of more than few $\mathrm{mm}$. The coated device was heated by applying $5 \mathrm{~V}$ with dry nitrogen gas flow at 423-453 K. The coated film was then baked in furnace at $443 \mathrm{~K}$ for $2 \mathrm{~h}$.

\subsection{Optical and X-ray diffraction studies}

A $\lambda-19$, UV-VIS Spectrophotometer (Perkin Elmer, USA) was used for measuring the optical properties of indium oxide thin films. The square of absorption coefficient $\left(\alpha^{2}\right)$ vs photon energy $(h v)$ curve for the indium oxide thin films grown on the cleaned glass surface is shown in figure 1. The direct band-gap of indium oxide thin film was found to be $3.62 \mathrm{eV}$ from the optical absorption studies. This was in good agreement with the value already reported by Manifacier et al (1981). A visible transmission $>75 \%$ with a film thickness of about $150 \mathrm{~nm}$ was obtained for indium oxide thin film. A moderately lower transparency and resistivity showed the existence of donor levels due to oxygen vacancies or in other words an excess of free metal, which makes it stable and sensitive gas sensor (Patel et al 1995).

Crystallinity of indium oxide thin film of thickness $300 \mathrm{~nm}$ was analysed by X-ray diffraction (X-pert MPD, Philips) method with $\mathrm{CuK}_{\alpha}$ radiation. The results of $\mathrm{X}$ -

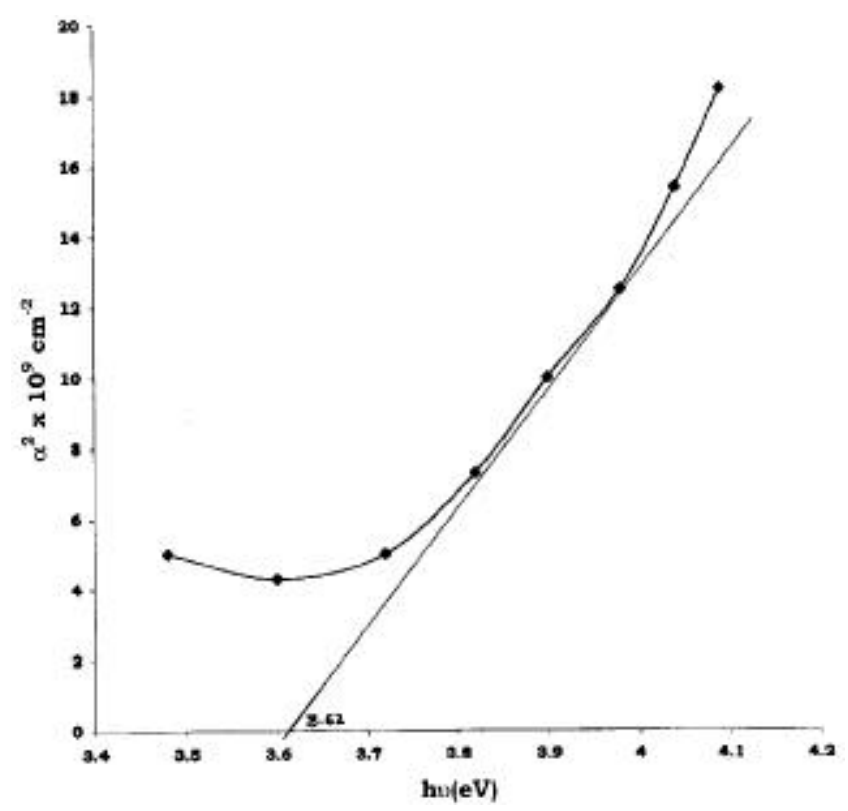

Figure 1. Absorption coefficient $\left(\alpha^{2}\right)$ vs photon energy $(h v)$ curve of indium oxide thin film. 


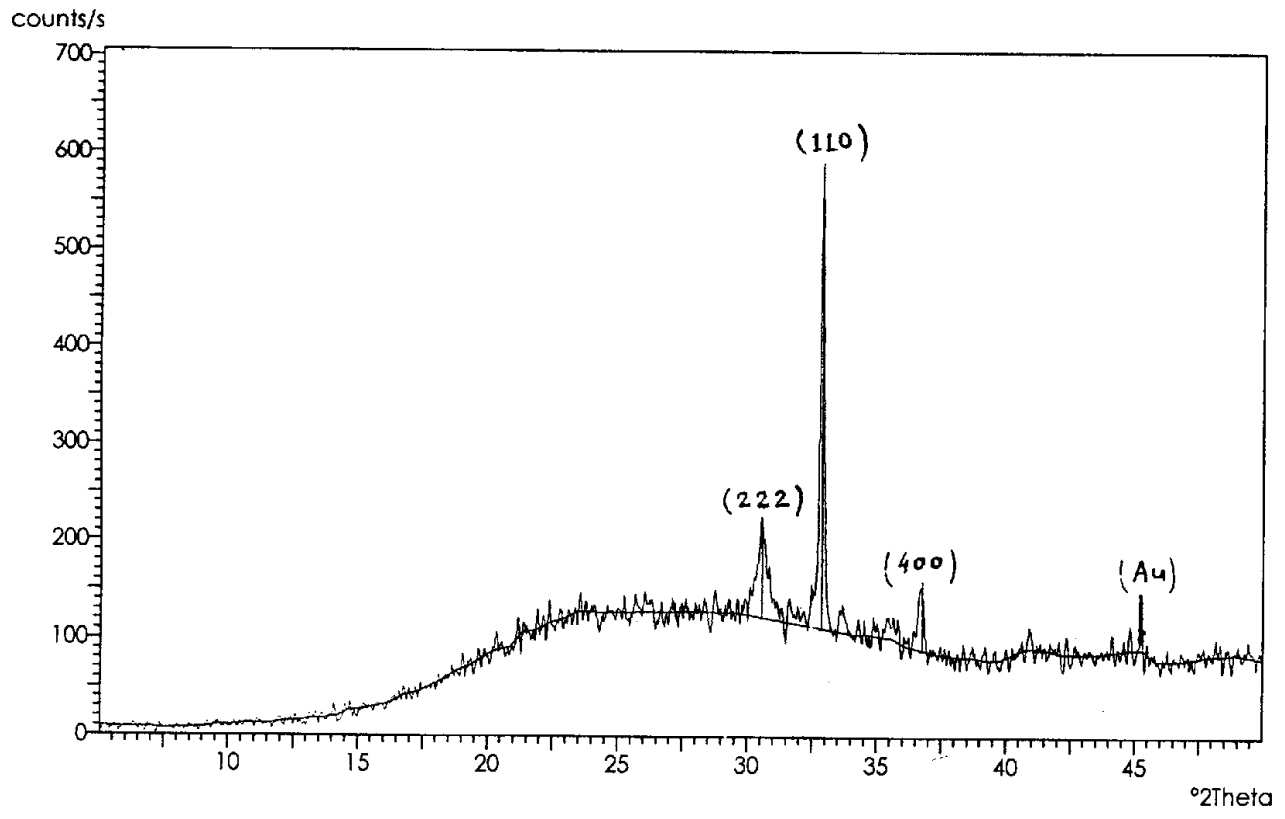

Figure 2. X-ray diffraction pattern of indium oxide thin film.

ray diffraction shown in figure 2 are similar to those reported by other workers (Radhakrishnan et al 1997) for indium oxide having cubic structure. The value of lattice constant, $10 \cdot 118 \AA$, agrees with the literature value reported for indium oxide. The film thus deposited and annealed at $700 \mathrm{~K}$ is a single-phase indium oxide. The gold particles which were deposited for the purpose of contact, dispersed as a few Angstrom layer over the sensing layer and exhibited a peak (111) at $45 \cdot 5^{\circ}$.

\subsection{Measurements}

The fabricated multipurpose sensor was mounted in a closed chamber into which desired amount of ammonia gas/ethanol vapour could be injected. A $6 \mathrm{~V}$ d.c. supply was applied to indium tin oxide heater encapsulated in poly(methyl methacrylate) (PMMA) over which insulating alumina substrate having gas sensing indium oxide layer with gold contacts was kept. The schematic diagram of this experiment is shown in figure 3 . The ratio of measured resistance before and after exposing the sensor surface to ammonia gas gives sensitivity,

$$
S=R_{\text {air }} / R_{\text {gas }},
$$

where $R_{\text {air }}$ is resistance in air and $R_{\text {gas }}$ the same for ammonia gas/ethanol vapour. Hall mobility and carrier density measurements were made using Van der Paw method.

\section{Results and discussion}

\subsection{Response to ammonia gas}

The experimental arrangement for sensitivity measurement is shown in figure 3 . The sensor is mounted on an

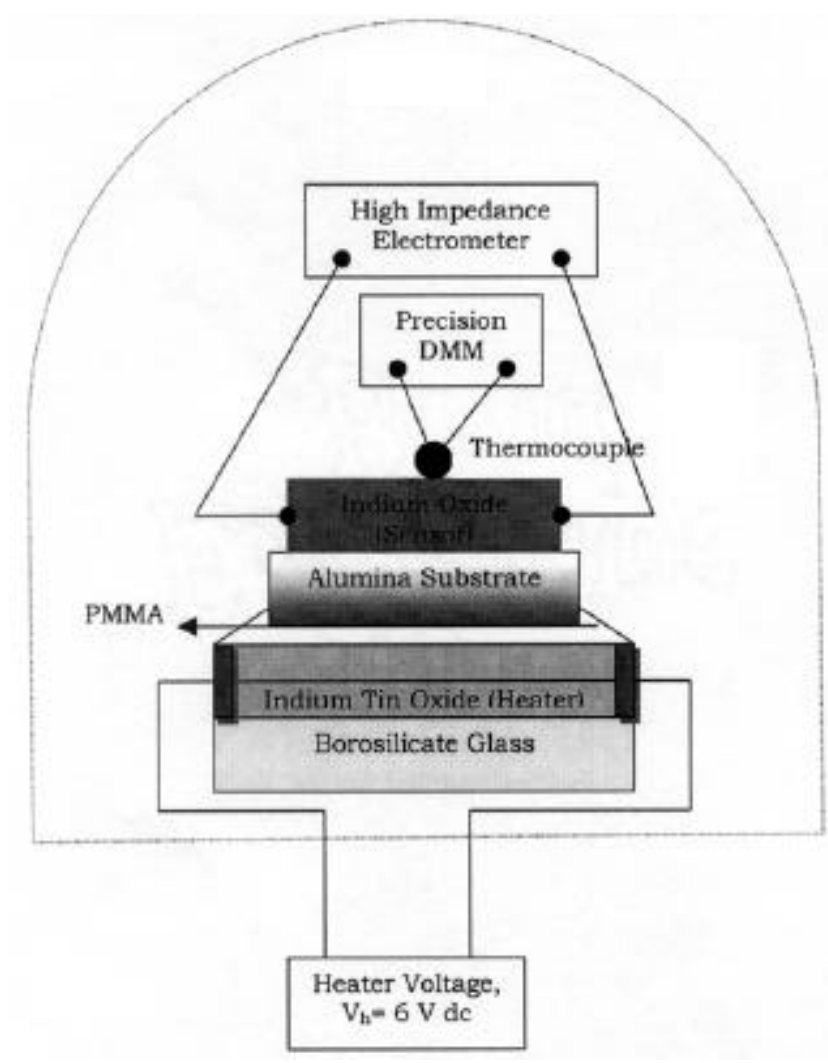

Figure 3. Schematic diagram of experimental setup.

encapsulated miniature indium tin oxide heater housed in a closed chamber. A $6 \mathrm{~V}$ d.c. bias was applied to the heater and temperature was measured using thermocouple and precision digital multimeter. The test gas i.e. ammonia, was passed through gas flow controller and by adjusting 
the flow rate of gas using flow controller, the concentration of ammonia in the test chamber could be changed. The conductance of the sensor was measured with digital electrometer (Keithley-614). The thermocouple measures a change in sensor temperature. The change in temperature, $T$ and resistance, $R$ of sensor were simultaneously measured when different concentrations of ammonia were introduced in test chamber (figure 4). It has been observed that the adsorption of ammonia increases the conductance of indium oxide thin films. The gas sensor was operated at different concentrations of ammonia in the temperature range $293-493 \mathrm{~K}$. At $473 \mathrm{~K}$, the sensitivity of the sensor was found saturated as shown in figure 5 . The ratio of measured resistance before and after exposing the sample surface to ammonia gives the value of sensitivity.

Figure 6 shows the plot of sensitivity vs operating temperature for two different thicknesses of indium oxide thin film. It was observed from the plot that thinner films exhibit higher sensitivity. It is concluded that thinner films of about $100 \mathrm{~nm}$ with low carrier concentration have better gas sensitivity than the thicker film with higher carrier concentration. The reason is that gas sensitivity is largest when the depletion region generated by chemisorbed oxygen extends through the sensor (Patel et al 1995).

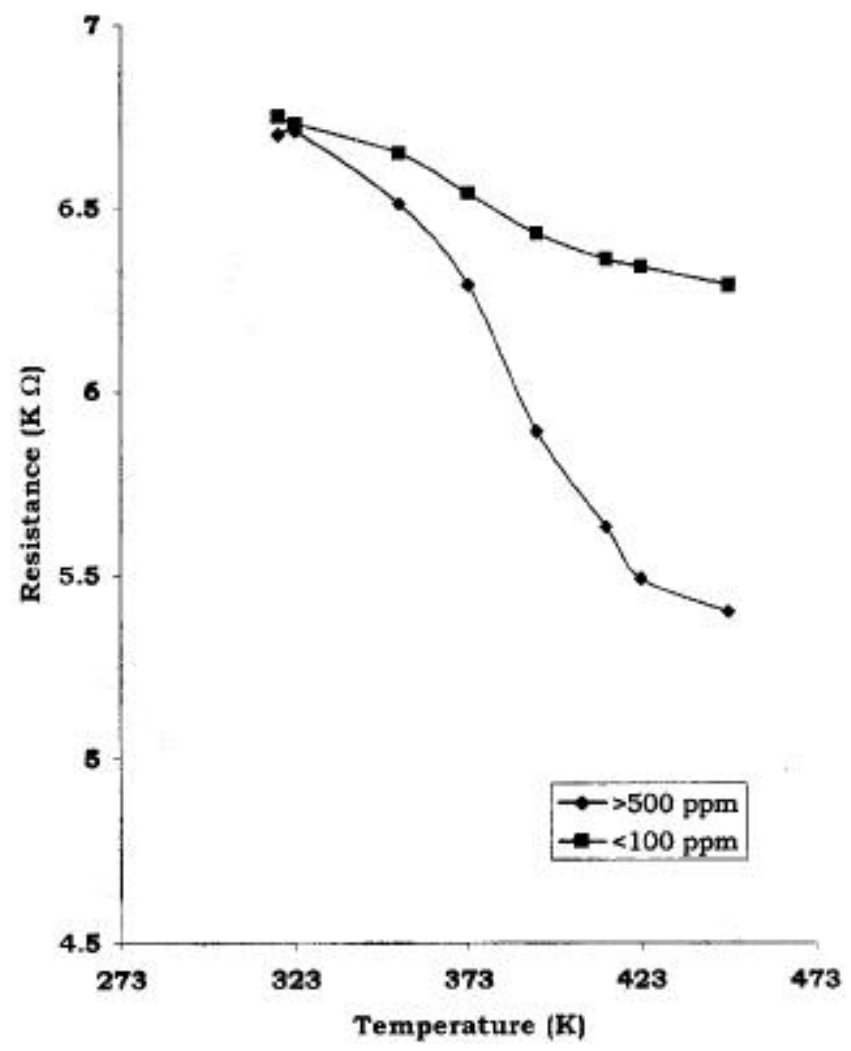

Figure 4. Resistance variation with temperature of indium oxide thin film when exposed to different concentrations of ammonia gas.
Actually, ammonia $\left(\mathrm{NH}_{3}\right)$ is a reducing gas for indium oxide. The resistance of $\mathrm{Au}$ modified $\mathrm{In}_{2} \mathrm{O}_{3}$ decreases on $\mathrm{NH}_{3}$ exposure at temperature between 323 and $448 \mathrm{~K}$. Earlier workers (Steffes et al 2001) have already observed the enhancement of sensitivity of indium oxide films due to the Au particle. They observed that when gold is deposited on selected metal oxide the sensor sensitivity increases. The sensors so far reported for ammonia are sensitive at elevated temperature of $675 \mathrm{~K}$. Interestingly in the present investigation the sensor is found to be sensitive at lower temperature of $448 \mathrm{~K}$. This may be due to the fact that $\operatorname{In}_{2} \mathrm{O}_{3}$ in its slightly non-stoichiometric form is a material that contains significant amount of localized centre with trapped electrons.

\subsection{Interfering effects of relative humidity}

The method presented to study the influence of relative humidity on the response of indium oxide thin film to ammonia gas consists of turning on and off the gas sensor between two well-defined temperatures. An indium oxide thin film was exposed to ammonia in air containing different amounts of humidity. The main purpose of this measurement was to check the sensitivity of sensor to ammonia and possible interfering effect of humidity. Figure 7 is a graph of sensitivity vs time of intermittent periodic heating at $323 \mathrm{~K}\left(T_{1}\right)$ and $473 \mathrm{~K}\left(T_{2}\right)$, respectively. At both the temperatures the heating is done for $3 \mathrm{~min}$. It was observed that the electrical conductance of a sensor changes by a few orders of magnitude when the tempera-

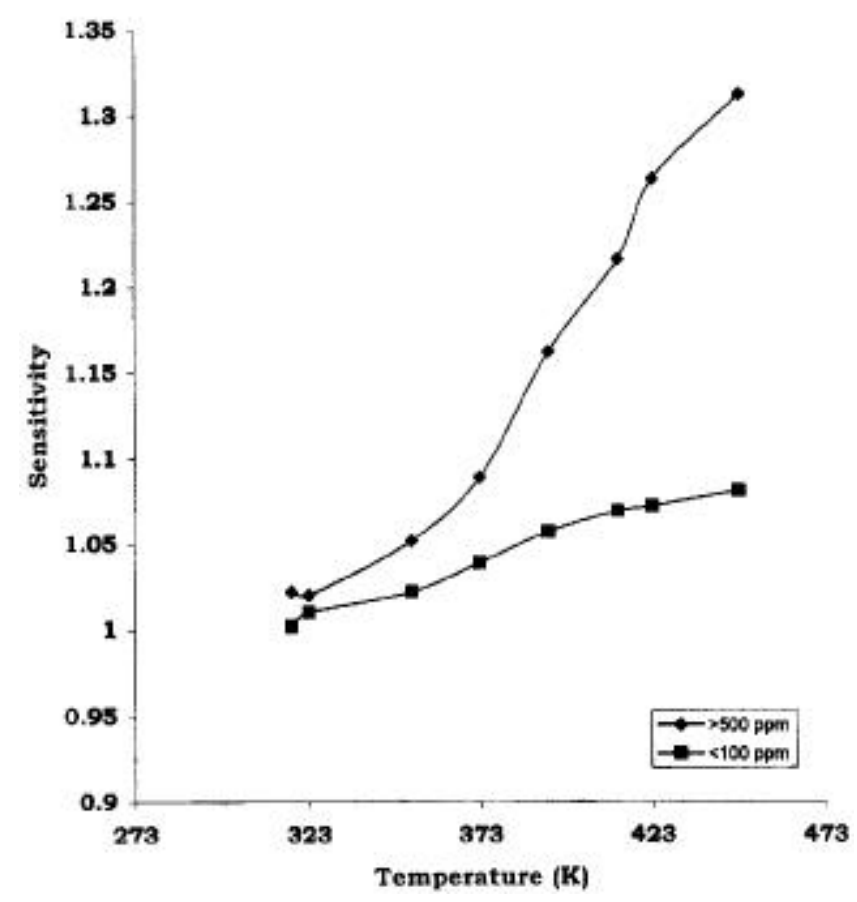

Figure 5. Sensitivity vs temperature of indium oxide thin films at different concentrations of ammonia gas. 
ture changes from $323-473 \mathrm{~K}$. The intermittent periodic heating of the sensor at two sets of temperatures in ammonia gave larger variations in conductance. The ratio of electrical conductance of the sensor when operating at a higher temperature and to that at lower temperature is unaffected by water vapour but is sensitive to ammonia. It is found that the detrimental effect of humidity on the sensing properties of indium oxide thin film gas sensors is almost negligible. It is also observed that at intermittent periodic heating mode of operation, both response and recovery times are long (8-10\%) (Patel et al 1994).

\subsection{Response to ethanol vapour}

The experimental arrangement for sensitivity measurement for ethanol vapour is shown in figure 3. Nitrogen gas was purged for $15 \mathrm{~min}$ to clean the sample environment. Ethanol was injected by micro-syringe into test chamber and sensing characteristics of the sensor was then observed. The change in electrical resistance of a sensor was measured by electrometer (Keithley). A typical injection of $0.4 \mathrm{ml}$ of ethanol corresponds to $10 \mathrm{ppm}$ as reported by Shurmer (1990). Before the measurement, the samples were preheated up to $473 \mathrm{~K}$ and then cooled up to $303 \mathrm{~K}$. The changes in sensor temperature, $\Delta T$ and resistance, $\Delta R$, of indium oxide based sensors of different thicknesses were simultaneously measured when they were exposed to fixed concentration of $400 \mathrm{ppm}$ ethanol vapours in test chamber and are shown in figure 8. It has been

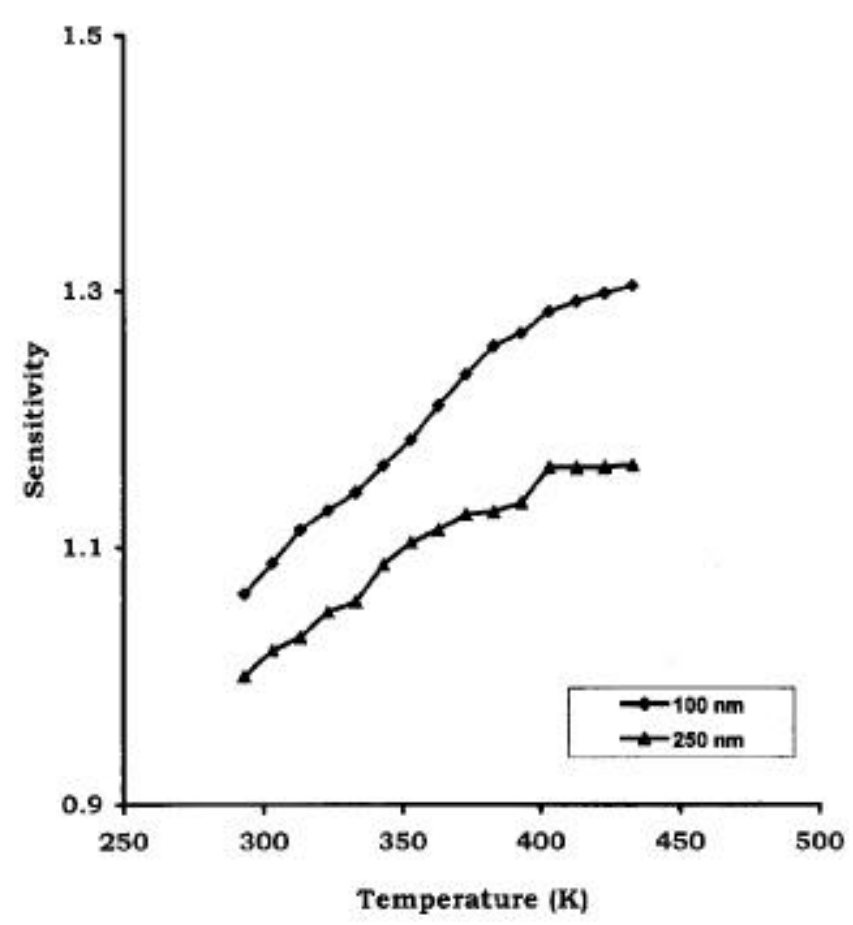

Figure 6. Sensitivity vs temperature at different thicknesses of indium oxide thin films for ammonia gas. observed that the adsorption of ethanol vapour increases the conductance of indium oxide thin films, operated at fixed concentration of $400 \mathrm{ppm}$ in the temperature range 293-393 K. Above $373 \mathrm{~K}$ the sensitivity of the sensors was found to be saturate. The time taken by the sensor for fall of resistance was considered as response time of a sensor. The ratio of measured resistance before and after

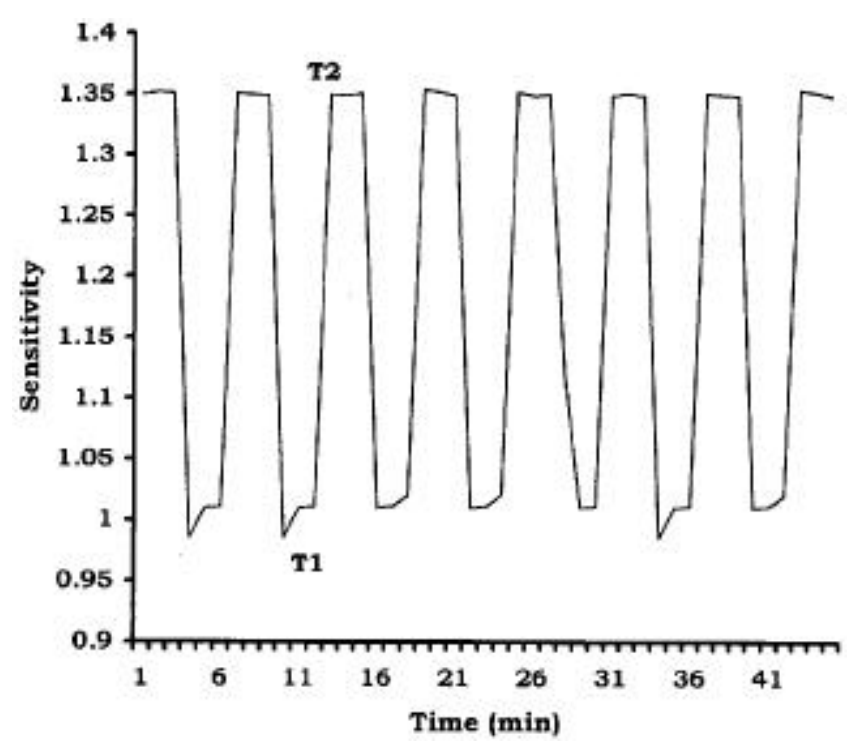

Figure 7. Intermittent periodic heating of ammonia sensor at $323 \mathrm{~K}$ and $473 \mathrm{~K}$.

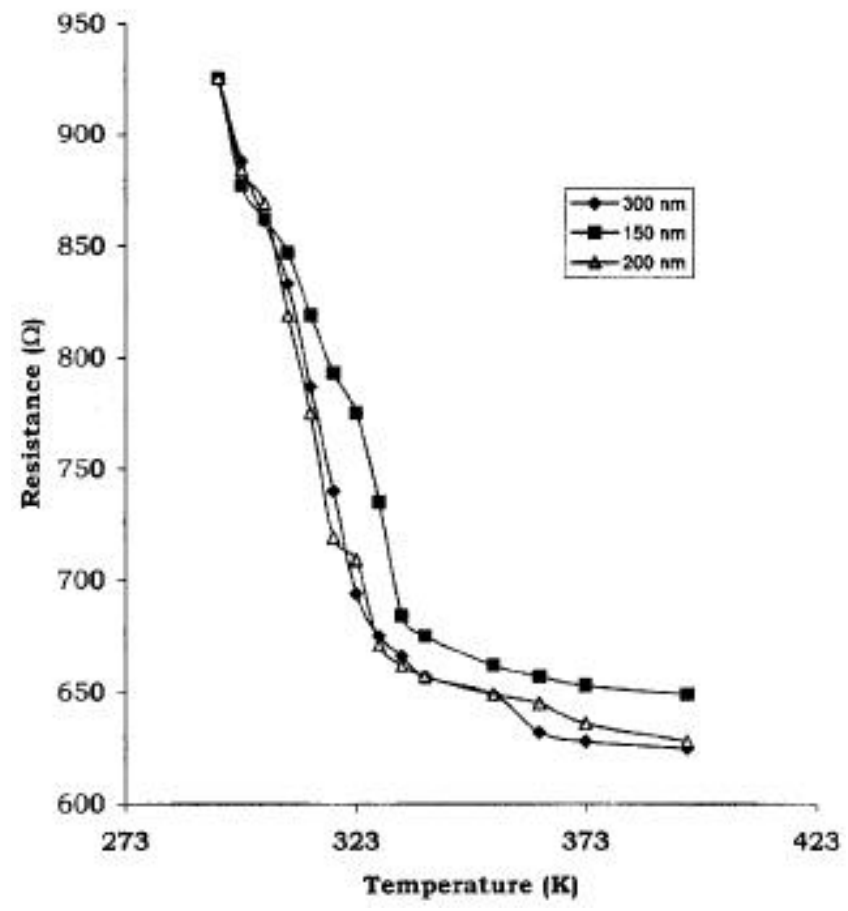

Figure 8. Resistance variation with temperature of indium oxide thin film gas sensor at $400 \mathrm{ppm}$ concentration of ethanol vapour for different thickness values. 
exposing the sample surface to ethanol vapour gives the value of sensitivity,

$$
S=R_{(\text {air })} / R_{(\text {gas })},
$$

as already have been stated earlier. This is shown in figure 9. Films of thickness ranging from 150-300 nm were investigated. However, results reported here are found to be independent of film thickness. The sensing properties were also studied at low concentrations of ethanol and it was found that gas is sensitive even at $300 \mathrm{ppm}$ of ethanol

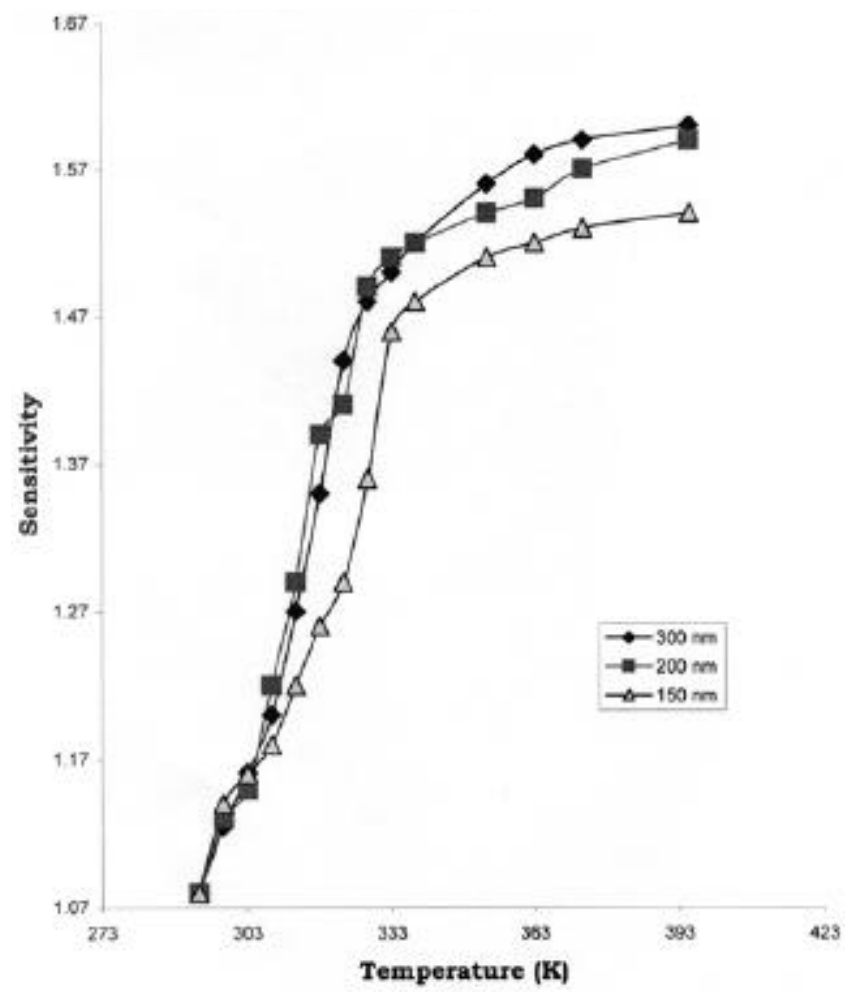

Figure 9. Gas sensitivity as a function of temperature for fixed concentration of $400 \mathrm{ppm}$ of ethanol vapours at various thicknesses. vapour. The response time is also fast. The results obtained by thermal evaporation technique are promising for the preparation of sensitive and low cost ethanol sensor operating at low temperatures. Magnetron sputtering technique can be used for fabrication of indium oxide gas sensor to detect variety of gases. By adding different dopants to the parent material, the sensitivity of indium oxide film can be increased.

\section{Block diagram of circuit}

Figure 10 is a schematic block diagram of the circuit. The ammonia sensor is placed in one arm of the bridge with resistance, $R_{\mathrm{c}}$, in the other arm. The other two resistances, $R_{\mathrm{a}}$ and $R_{\mathrm{b}}$, form the opposite arms of bridge to balance the potential. At reference condition, the bridge is balanced to a constant potential. When the circuit is placed to sense the ammonia, change in resistance is observed. A reducing molecule adsorbed at the surface of indium oxide, acts as a surface donor injecting the electrons into the semiconductor. According to the adsorption of ammonia gas on the surface the resistance varies in the bridge. The unbalanced resistance value in the bridge causes the potential change with respect to reference potential. The observed change in potential value is the proportional quantity to the change in concentration of ammonia gas on the surface due to absorption process. The ICLF353, dual operational amplifier in a single package instrumentation amplifier is employed for measurement purpose. It is constructed as voltage follower with high input impedance so as to avoid the loading effect in the circuit. The output part of the circuit is a display, which is next to the instrumentation amplifier. The LEDs are used to detect signals of the increasing and decreasing concentrations of ammonia. The change in decade concentration of ammonia is observed through glowing of LED. LM3914, LED driver is used to drive the weak signals received from the amplifier up to the display. This chip

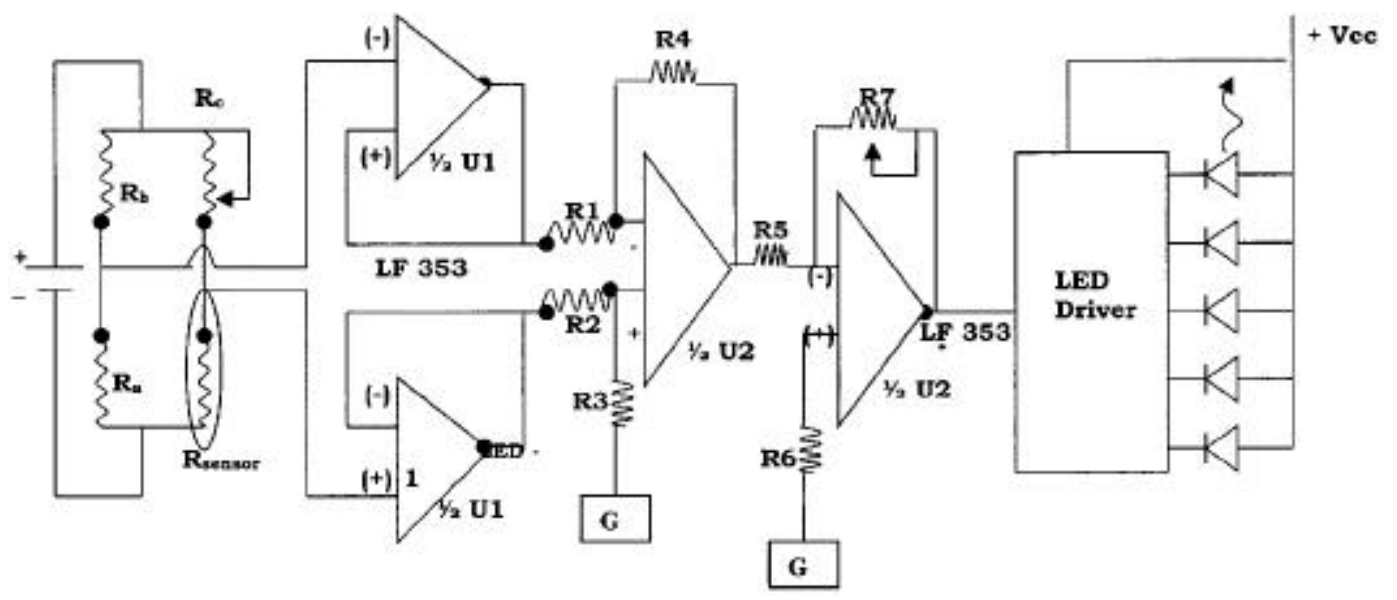

Figure 10. Schematic circuit for ammonia sensor. 


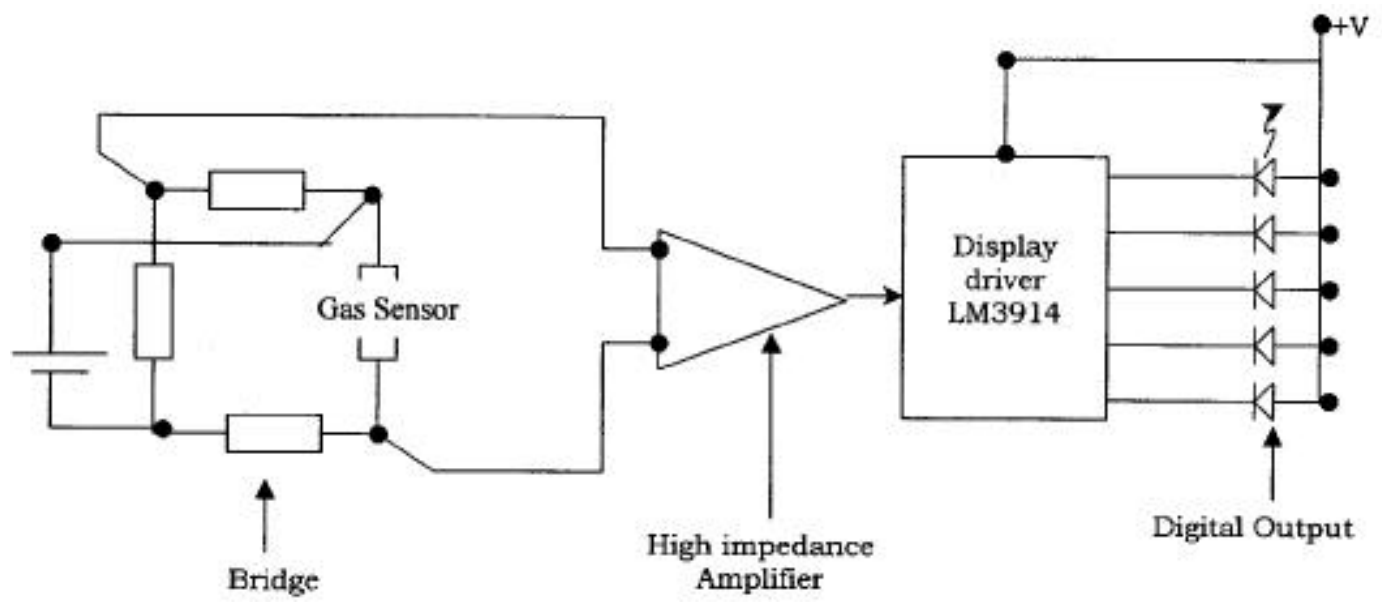

Figure 11. Block diagram of electronic sensing of ethanol vapour.

has the capacity to drive 10 LEDs simultaneously providing a linear analog display.

The block diagram of electronic sensing alarm for ethanol vapours is shown in figure 11. This circuit also consists of a bridge, high impedance amplifier and an LED display. The change in concentration causes a change in resistance into the bridge. The weak signals are detected and amplified by the high impedance amplifier. These amplified signals are then fed to the same display driver used in ammonia sensing i.e. LM3914, providing a linear analog display of the ethanol vapour absorbed on the surface.

\section{Conclusions}

Indium oxide thin film sensor for ammonia gas and ethanol vapour has been fabricated and indium tin oxide thin films encapsulated in PMMA has been used as miniature heater. The films have been characterized by X-ray diffraction, optical and electrical studies. The sensitivity of the sensor for an ammonia gas and ethanol vapour has been obtained. The presence of humidity does not interfere with sensitivity. The sensitivity has been found to be independent of thickness of the films for ethanol vapours, while it is dependent on thickness for the ammonia gas. It has been observed that for ammonia gas the lower the thickness the higher the sensitivity. Work on encapsulation of ITO miniature heaters operating at higher temperatures using poly (methyl methacrylate) and polyvinylidene fluoride (PVDF) is in progress.

\section{Acknowledgement}

We are thankful to the Sophisticated Instrumentation Centre for Applied Research and Testing (SICART), Vallabh Vidyanagar, for providing the necessary facilities.

\section{References}

Echigoya J, Kato S and Enoki H 1992 J. Mater. Sci. -Mater. Electron. 3168

Ertl G 1982 Chem. and phys. solid surf. (FL: CRC Press) Vol. III

Fritzche H, Pashmakov B and Claflin B 1994 Solar Energy Mater. Solar Cells 32383

Gagaoudakis E, Bender M, Douloufakis E, Katsarakis N, Natsakou E, Cimalla V and Kiriakidis G 2001 Sens. \& Act. B80 155

Girtan M and Rusu G I 1999-2000 Analele Stintifice Ale Universitatii, Al. I. Cuza Din Iasi Tomul XLV-XLVIs Fizica Starii Condenstate pp 166-172

Gopel W 1985 Mess. 52 47, 92, 175

Hamberg H, Granquist C G, Berggen K F, Sernelieus B E and Engstron L 1984 Phys. Rev. B30 3240

Kanefusa S, Nitta M and Harodome M 1983 Anal. Chem. Symp. Ser. 1784

Kim H, Horwitz J S, Pique A, Gilmore C M and Chrisey D B 1990 Appl. Phys. Suppl. Mater. Sci. \& Process. A69 S447

Manifacier J C, Szepessy L, Bress J F, Perotin M and Stuck R 1979 Mater. Res. Bull. 14 109, 163

Manifacier J C, Fillard J P and Bind J M 1981 Thin Solid Films 7767

Moseley P and Williams E 1990 Sens. \& Act. 81113

Nanto H, Minami T and Takata S 1986 J. Appl. Phys. 60 482

Nelli P and Sberveglieri G 1994 in Sensors for domestic applications (eds) Arnaldo D'Amico and Giorgio Sberveglieri (Proceeding of the Ist European school on sensors) (Leece, Italy: Castro Marina)

Obayashi H, Sakurai Y and Geo T 1976 J. Solid State Chem. 17 299

Ogawa H, Abe A, Nishikawa M and Hayaka S 1981 J. Electrochem. Soc. 1282020

Oomman K, Varghese L and Malhotra K 1998 Sens. \& Act. B53 19

Patel N G and Lashkari B H 1992 J. Mater. Sci. 273026 
Patel N G, Makhija K K and Panchal C J 1994 Sens. \& Act. B21 193

Patel N G, Makhija K K, Panchal C J, Dave D B and Vaishnav V S 1995 Sens. \& Act. B23 49

Radhakrishnan C, Subramanyam T K, Uthanne S and Srinivaala Naidu B 1997 Bull. Indian Vac. Soc. 2817

Raju H R and Rao C N R 1991 Sens. \& Act. B3/4 365
Rao C N R, Raju A R and Vijaymohan K 1992 New materials (New Delhi: Narosa Publishing House) Ch. 1, pp 1-37

Sears W M, Colbow K and Conradori F 1989 Sens. \& Act. 19333 Shurmer H V 1990 Sens. \& Act. B1 48

Steffes H, Imawan C, Solzbacher F and Obermeier E 2001 Sens. \& Act. B78 106

Tadashi Takada 1998 Sens. \& Act. B52 45 